\title{
Vascular Endothelial Growth Factor-1 Level and Functional Neurologic Recovery after Ischemic Hemispheric Stroke
}

\author{
Svetlana A Medvedkova ${ }^{1}$ and Alexander E Berezin²* \\ ${ }^{1}$ Department of Nervouse Diseases, State Medical University, Mayakovsky av., Zaporozhye, Ukraine \\ ${ }^{2}$ Internal Medicine Department, State Medical University, Mayakovsky av., Zaporozhye, Ukraine
}

\begin{abstract}
Background: Vascular endothelial growth factor-1 (VEGF-1) acts as endogeneous angiopoetic and neuroprotective factor. The aim of the study to investigate an interrelationship between VEFG-1 elevation and neurologic recovery in short-term period among patients with ischemic hemispheric stroke.

Methods: Seventy eight patients with ischemic hemispheric stroke were included in the study. Neurological impairment at presentation was assessed according National Institute of Health Stroke Scale. The Barthel Index and the modified Rankin Scale (mRS) were used to assess functional disability. Concentrations of VEGF-1 were measured at baseline using ELISA laboratory kit.

Results: The total prevalence of hypertension was $100 \%$ (78 patients). $79.5 \%$ patients had dyslipidemia, $18 \%$ were diabetic, $15.4 \%$ of the total population was smokers. Among study patient population a total of $19.2 \%$ subjects had a past history of ischemic heart diseases in their families and $11.5 \%$ had experienced a previous ischemic heart episode. Within 6 month after baseline the favorable neurologic recovery defined as mRS $\leq 2$ was found in 64 patients $(82.1 \%)$. We calculated Modified Rankin Score $\geq 3$ in 14 patients (17.9\%). Depending on Modified Rankin Score at 6 month $(\leq 2$ and $\geq 3)$ we have divided all patients into two sub-groups respectively. We found a higher prevalence of subjects with dyslipidemia $(P=0.044)$, diabetes mellitus $(P=0.024)$, smokers $(P=0.025)$ and as well as those with ischemic heart disease in their family histories $(P=0.026)$ and previous ischemic heart episode $(P=0.032)$ in subgroup with $\mathrm{mRS} \geq 3$. At baseline VEGF-1 concentration in entire patient cohort was $348.55 \mathrm{pg} /$ $\mathrm{mL}$ (IQR=241.70-493.80 pg/mL). We found higher VEGF-1 level in $\mathrm{mRS} \geq 3$ vs $\mathrm{mRS} \leq 2$ population sub-groups $(P<0.05)$. Using multivariate Cox regression analysis we reported that VEGF-1 (increased per $125 \mathrm{pg} / \mathrm{mL}$ ) and type two diabetes mellitus (present versus absent) were found as independent predictors for favorable neurologic recovery.

Conclusion: In Conclusion, We found that VEGF-1 (increased per $125 \mathrm{pg} / \mathrm{mL}$ ) measured at baseline positively predicts improved neurologic recovery at six month among subjects with acute ischemic stroke who were not enrolled for invasive or thrombolytic strategy. Type two diabetes mellitus (present versus absent) were found as independent predictor for favorable neurologic recovery in follow up period.
\end{abstract}

Keywords: Acute ischemic stroke; Vascular endothelial growth factor-1; Neurologic recovery

Abbreviations: BMI: Body Mass Index; LDL: Low Density Lipoproteins; HDL: High Density Lipoproteins; LAAS: Large Artery Atherosclerosis; LAC: Lacunar Infarct; UDE: Stroke of Undetermined Etiology

\section{Introduction}

Stroke remains a major public health problem worldwide, although stroke mortality has been declining since the early 20th century [1]. These significant improvements in stroke outcomes are concurrent with cardiovascular $(\mathrm{CV})$ risk factor control interventions, more widely use of revascularization procedure technologies, post-stroke rehabilitation, etc. $[2,3]$. Because of the heterogeneity of stroke, it is uncertain whether other stroke subtypes should be considered to be at the same high levels of risk [3]. Probably, biomarker-guided therapy of stroke might be useful instrument to optimize CV risk factor and stroke treatment scheme and post-stroke rehabilitation. However, there are uncertainties surrounding the optimal management of patients with ischemic stroke under biomarker control to improve neurological recovery $[4,5]$. Therefore, the impact of biomarker in risk prediction of CV outcome cluster and post-stroke rehabilitation is under investigations [6]. Vascular endothelial growth factor-1 (VEGF-1) as a ligand for alpha-5 / beta-1 integrin is a key factor in angiogenesis that may not only stimulate vasculogenesis via mobilizing of endothelial progenitor cells, but it is able to regulate production of several endogeneous pro-angiogenic factors, i.e., insulin-like growth factor-1, platelet-derived growth factor [7-9]. Most biological effects of VEGF are mediated via two receptor tyrosine kinases, i.e., VEGFR1 and VEGFR2. They contribute transcriptional and post-translational signaling pathways respectively [10]. There is evidence that VEGF-1 stimulates phosphoinositide 3' kinase/AKT/endothelial nitric oxide synthase pathway and suppresses reactive oxygen species overproduction, accumulation of advanced glycation end products [11]. Therefore, VEGF-1 has neurotrophic effect on neuronal and glial cells in culture and in vivo, and may stimulate the proliferation and survival of neural stem cells [10]. Overall, VEGF-1 acts as trophic factor with angiopoetic and neuroprotective properties

${ }^{*}$ Corresponding author: Alexander E Berezin, Professor, MD, PhD, Internal Medicine Department, State Medical University, 26, Mayakovsky av, Zaporozhye, Ukraine, Tel: +380612894585; E-mail: dr_berezin@mail.ru

Received September 23, 2015; Accepted September 30, 2015; Published October 07, 2015

Citation: Medvedkova SA, Berezin AE (2015) Vascular Endothelial Growth Factor-1 Level and Functional Neurologic Recovery after Ischemic Hemispheric Stroke. Neurochem Neuropharm Open Access 1: 102.

Copyright: () 2015 Medvedkova SA, et al. This is an open-access article distributed under the terms of the Creative Commons Attribution License, which permits unrestricted use, distribution, and reproduction in any medium, provided the original author and source are credited. 
$[10,12]$. Elevated level of VEGF-1 was recently found in patients after acute stroke and they were demonstrated predictive value for recurrent $\mathrm{CV}$ events in long-term follow up [13,14]. Although a neurologic deficit of sudden onset conforming to a vascular territory is a clear clinical indication in acute ischemic stroke, the predictive role of VEFG-1 for neurologic recovery in short-term period is still not clear. The aim of the study: to investigate an interrelationship between VEFG-1 elevation and neurologic recovery in short-term period among patients with ischemic hemispheric stroke.

\section{Methods}

\section{Study population}

This prospective, observational and population study took place over a six month period. A sample size was estimated considering an alpha error of $5 \%$, a statistical power of $95 \%$ and an estimated loss ratio of $5 \%$. A total of 78 patients, who had previously defined ischemic hemispheric stroke and who were not enrolled for further intravenous thrombolysis due to technical reasons, were included in the study. Ischemic hemispheric stroke was defined according criteria of Stroke Council of the American Heart Association/American Stroke Association [4]. All the patients have given their voluntary informed written consent for participation in the study. The study was approved by the local ethics committee of State Medical University, Zaporozhye, Ukraine. The study was performed in conformity with the Declaration of Helsinki.

\section{Clinical and demographic data}

The clinical and demographic data was collected in regard to their case history: age, weight, height, family history, personal information, prior atherosclerotic vascular disease (defined as prior coronary heart disease, cerebral vascular disease), hypertension (defined as blood pressure $>140$ / $90 \mathrm{~mm} \mathrm{Hg}$ ), diabetes mellitus (DM), dyserlipemia, and having a smoking habit. Current smoking was defined as consumption of one cigarette daily for three months. Anthropometric measurements were made using standard procedures.

\section{Neurological status}

Neurological impairment at presentation was assessed by National Institute of Health Stroke Scale (NIHSS) (National Institute of Health Stroke Scale) [4]. The Barthel Index [15] and the modified Rankin Scale [16] were used to assess functional disability.

\section{Contrast-enhanced computer spiral tomography}

To define brain infarct and exclude intracranial hemorrhage among all patients suspected ischemic stroke at baseline contrast-enhanced computer spiral tomography (CT) was performed on a "Somatom Spirit" scanner (Siemens, Germany). Nonionic contrast "Omnipak" (Amersham Health, Ireland) was used. The acute ischemic stroke type was classified according to the TOAST classification: 1) large artery atherosclerosis (LAAS); 2) cardioembolic infarct (CEI); 3) lacunar infarct (LAC); 4) stroke of other determined etiology (ODE); 5) stroke of undetermined etiology (UDE) [17].

\section{Calculation of glomerular filtration rate}

Glomerular filtration rate (GFR) was calculated with CKD-EPI formula [18].

\section{Measurement of circulating biomarkers}

To determine circulating biomarkers, blood samples were collected at baseline in the morning (at 7-8 AM) into cooled silicone test tubes wherein $2 \mathrm{~mL}$ of $5 \%$ Trilon B solution were added. Then they were centrifuged upon permanent cooling at $6,000 \mathrm{rpm}$ for 3 minutes. Plasma was collected and refrigerated immediately to be stored at a temperature $-70^{\circ} \mathrm{C}$. Concentrations of VEGF-1 were measured by ELISA at baseline using laboratory kits produced by Bioscience (USA). All determinations were done by duplicate. Concentrations of total cholesterol (TC), cholesterol of high-density lipoproteins (LDL-C), and cholesterol of high-density lipoproteins (HDL-C) at baseline were measured by enzymatic colorimetric method according standardized methodology on Beckman Synchron LX20 chemistry analyzer.

\section{Statistical analysis}

The statistical calculations were made with the IBM statistical package Statistical Package for Social Sciences (SPSS), version 20. The normal distribution test was carried out with the Shapiro-Wilk test. The continuous variables followed a normal and abnormal distribution were expressed as mean (M) and average standard deviation (SD) and median $(\mathrm{Me})$ and interquartile range (IQR). Categorical variables were expressed with frequencies in percentages. To compare the qualitative variables the $\mathrm{Chi}^{2}$ test and the Fisher exact test were applied. The analysis of the two independent samples was made with the Student's t-test (normal distribution) or the Mann-Whitney U test. Age- and sex adjusted univariate and multivariate regression analysis for neurologic recovery defined as Modified Rankin Score $\leq 2$ at 6 month. In the Cox regression model the significance of Odds Ratios (OR) was tested on the basis of Wald statistics. A two-tailed probability value of $<0.05$ was considered as significant.

\section{Results}

The general characteristics of the clinical population are set forth in Table 1 . The total prevalence of hypertension was $100 \%$ (78 patients). $79.5 \%$ were dyslipemic, $18 \%$ were diabetic, $15.4 \%$ of the total population were smokers. A total of $19.2 \%$ had a past history of ischemic heart diseases in their families and $11.5 \%$ had experienced a previous ischemic heart episode. According TOAST criteria LAAS was defined in $47.4 \%$ patients, LAC was found in y $41.0 \%$, and UDE was detected in $11.6 \%$ subjects. Median of brain infarct volume at baseline defined using contrast-enhanced spiral CT was $3.52 \mathrm{~cm}^{3}\left(1.47-8.51 \mathrm{~cm}^{3}\right)$. Right side and left side localization of infarct was detected in $37.2 \%$ and $62.8 \%$ respectively. At baseline mediana of NIHSS score and Modified Rankin Score were 5.00 and 3.00 respectively. All patients were treated with optionally strategy (ACE inhibitors or angiotensin-II receptor antagonists, antiplatelets, statins) within 6 month. In 6 month after baseline the favorable neurologic recovery defined as Modified Rankin Score $\leq 2$ was found in 64 patients $(82.1 \%)$. In contrast, Modified Rankin Score $\geq 3$ was calculated in 14 patients (17.9\%). Depending on Modified Rankin Score at 6 month $(\leq 2$ and $\geq 3$ ) we divided all patients into two groups respectively. Both groups were matched age, male sex, $\mathrm{CV}$ risk factors, BMI, brain infarct volume, types of ischemic stroke, systolic and diastolic blood pressure, biochemistry data, and treatment strategy.

In our analysis of Modified Rankin Score $\geq 3$ vs Modified Rankin Score $\leq 2$ population sub-groups, we observed a higher prevalence of dislipidemic subjects $(\mathrm{P}=0.044)$, diabetics $(\mathrm{P}=0.024)$, smokers $(\mathrm{P}=0.025)$ and as well as those with ischemic heart disease in their family histories $(\mathrm{P}=0.026)$ and previous ischemic heart episode $(\mathrm{P}=0.032)$ At baseline VEGF-1 concentration in entire patient cohort was $348.55 \mathrm{pg} / \mathrm{mL}(\mathrm{IQR}=241.70-493.80 \mathrm{pg} / \mathrm{mL})$. There was significant difference between VEGF-1 in both population sub-groups at baseline 


\begin{tabular}{|c|c|c|c|c|}
\hline & \multirow[t]{2}{*}{ Entire patient cohort at baseline $(n=78)$} & \multicolumn{2}{|c|}{ Both patient subgroups at 6 month } & \multirow{2}{*}{$P$ value } \\
\hline & & Modified Rankin Score $\leq 2(n=64)$ & Modified Rankin Score $\geq 3(n=14)$ & \\
\hline Age, years & $58.5 \pm 0.9$ & $57.6 \pm 0.6$ & $59.3 \pm 0.5$ & 0.99 \\
\hline Male, $n(\%)$ & $58(74.3 \%)$ & $47(73.4 \%)$ & $11(78.5 \%)$ & 0.86 \\
\hline Hypertension, n (\%) & $78(100 \%)$ & $64(100 \%)$ & $14(100 \%)$ & 1.0 \\
\hline Dyslipemia, $\mathrm{n}(\%)$ & $62(79.5 \%)$ & $50(78.1 \%)$ & $12(85.7 \%)$ & 0.044 \\
\hline Type two diabetes mellitus, $n(\%)$ & $14(18 \%)$ & $8(12.5 \%)$ & $6(42.9 \%)$ & 0.024 \\
\hline Current smoking status, n (\%) & $12(15.4 \%)$ & $7(11.0 \%)$ & $5(35.7 \%)$ & 0.025 \\
\hline Past history of ischemic heart diseases & $15(19.2 \%)$ & $9(14.0 \%)$ & $6(42.9 \%)$ & 0.026 \\
\hline Previous ischemic heart episode & $9(11.5 \%)$ & $5(7.8 \%)$ & $4(28.6 \%)$ & 0.032 \\
\hline LAAS, n (\%) & $37(47.4 \%)$ & $30(46.9 \%)$ & $7(50.0 \square)$ & 0.34 \\
\hline LAC, n (\%) & $32(41.0 \%)$ & $26(40.6 \%)$ & $6(42.8 \%)$ & 0.72 \\
\hline UDE, n (\%) & $9(11.6 \%)$ & $8(12.5 \%)$ & $1(7.1 \%)$ & 0.24 \\
\hline Brain infarct volume, $\mathrm{cm}^{3}$ & $3.52(1.47-8.51)$ & $3.40(1.14-6.59)$ & $4.82(3.25-14.33)$ & 0.18 \\
\hline Left-side localization, n (\%) & $49(62.8 \%)$ & $40(63.0 \%)$ & $9(64.2 \%)$ & 0.98 \\
\hline Right-side localization, n (\%) & $29(37.2 \%)$ & $24(37.0 \%)$ & $5(35.8 \%)$ & 0.92 \\
\hline NIHSS score at baseline & $5.00(3.25-7.75)$ & $4.00(3.00-7.00)$ & $9.00(7.00-9.75)$ & 0.12 \\
\hline Modified Rankin Score at baseline & $3.00(2.00-3.00)$ & $3.00(2.00-3.00)$ & $4.00(3.25-4.00)$ & 0.68 \\
\hline $\mathrm{BMI}, \mathrm{kg} / \mathrm{m}^{2}$ & $26.95(24.49-30.47)$ & $27.05(24.62-30.47)$ & $26.09(23.75-29.23)$ & 0.72 \\
\hline Systolic BP, mm Hg & $135(125-150)$ & $130(120-150)$ & $140(130-140)$ & 0.88 \\
\hline Diastolic BP, mm Hg & $82(71-89)$ & $83(70-87)$ & $80(80-95)$ & 0.86 \\
\hline Glucose, $\mathrm{mmol} / \mathrm{L}$ & $4.70(4.31-5.45)$ & $4.65(4.30-5.45)$ & $5.00(4.51-6.15)$ & 0.68 \\
\hline Creatinine, $\mathrm{mmol} / \mathrm{L}$ & $0.10(0.09-0.11)$ & $0.10(0.09-0.11)$ & $0.10(0.09-0.11)$ & 1.00 \\
\hline GFR, $\mathrm{mL} / \mathrm{min} \times 1.78 \mathrm{~m}^{2}$ & $81.00(72.25-95.75)$ & $82.50(73.75-97.25)$ & $74.00(68.25-82.50)$ & 0.78 \\
\hline Total cholesterol, $\mathrm{mmol} / \mathrm{L}$ & $6.52(6.00-7.00)$ & $5.66(4.54-6.55)$ & $4.25(3.67-6.26)$ & 0.46 \\
\hline LDL cholesterol, mmol/L & $4.44(3.90-4.80)$ & $6, .45(6.08-6.93)$ & $7.60(5.75-8.30)$ & 0.48 \\
\hline HDL cholesterol, mmol/L & $1.15(1.08-1.30)$ & $4.34(3.95-4.65)$ & $5.40(3.85-6.30)$ & 0.72 \\
\hline Triglycerides, mmol/L & $1.81(1.40-2.36)$ & $1.18(1.04-1.35)$ & $1.10(1.10-1.20)$ & 0.77 \\
\hline
\end{tabular}

Table 1: General characteristic of the patients enrolled in the study.

(Figure 1). Higher VEGF-1 level was found in Modified Rankin Score $\geq 3$ vs Modified Rankin Score $\leq 2$ population sub-groups $(\mathrm{P}<0.05)$. Age- and sex adjusted univariate regression analysis has shown that VEGF-1 were determined as predictors for neurologic recovery defined as Modified Rankin Score $\leq 2$ at 6 month (Table 2). However, after including in multivariate regression model all variables with $\mathrm{p}$ value $<0.2$, we found that VEGF-1, dyslipidemia, and type two diabetes mellitus has remained independent predictors for favorable neurologic recovery. Table 3 shows the results of the Multivariate Cox regression analysis for favorable neurologic recovery (Modified Rankin Score $\geq 3$ vs Modified Rankin Score $\leq 2$ ). As one can see, VEGF-1 (increased per $125 \mathrm{pg} / \mathrm{mL}$ ) and type two diabetes mellitus (present vs absent) were found as independent predictors for favorable neurologic recovery.

\section{Discussion}

Atherosclerosis is the main pathophysiological mechanism in patients with non-cardioembolic stroke, including large- and small-vessel disease. While an induction of VEGF-1 on endogenous neurogenesis and angiogenesis is known, the innate mechanisms of a therothrombotic-related evolution of injury and activated endogenous repair mechanisms are not fully understood. In the study we reported that increased baseline concentration of VEGF-1 may predict favorable neurologic recovery at 6 month defined as Modified Rankin Score $\leq 2$ in patients with acute ischemic stroke. There are large bodies of evidences regarding sex, age, stroke etiology, behavioral, genetic, neurological deficiency, and CV predictors of faster recovery of function following a stroke [19-21]. On the one hand, for most acute ischemic stroke patients the recovery of function during the first six months after stroke is $70 \%$ of the maximum possible [5]. On the other hand, the recovery after stroke is limited extremely deep and severe paresis, which is independent predictor of movement recovery in patients after stroke
[22,23]. Nevertheless, the reliable predictors of this response are not fully determined. Our findings indicate that increased circulating level of peak VEGF-1 in acute stroke patients without severe disability might be discussed a marker for faster recovery of function within 6 month. This biomarker associated with low grade Modified Rankin Score at 6 month, while type two diabetes mellitus required to be taken into consideration as the second independent predictor. In is noted that the results were receive using age- and sex adjusted multivariable analysis that explain lack of serious impact of these important variables on risk of faster recovery. We did not find similar data in recently published reports. However, we believe that more investigations need to explain these findings and clear the role and underlying molecular effect of exaggerated level of VEGF-1 in ischemic stroke subjects. Because of a significant recovery in impaired functional domains can be expected during neuropsychological rehabilitation, treatment strategy based on measurements of circulating VEGF-1 could be appeared attractive. Whether measurement of circulating VEGF-1 may help to predict cognition impairment is not fully understood and it is required more scrutinizes.

\section{Conclusion}

For subjects with acute ischemic stroke who were not enrolled for invasive or thrombolytic strategy, VEGF-1 (increased per $125 \mathrm{pg} / \mathrm{mL}$ ) measured at baseline positively predicts improved neurologic recovery at six month. In contrast, type two diabetes mellitus (present vs absent) were found as independent predictor for favorable neurologic recovery in follow up.

\section{Limitations of the Study}

This study has some limitations. We relied on clinical data to rule 
Citation: Medvedkova SA, Berezin AE (2015) Vascular Endothelial Growth Factor-1 Level and Functional Neurologic Recovery after Ischemic Hemispheric Stroke. Neurochem Neuropharm Open Access 1: 102.

$\mathrm{P}<0.05$

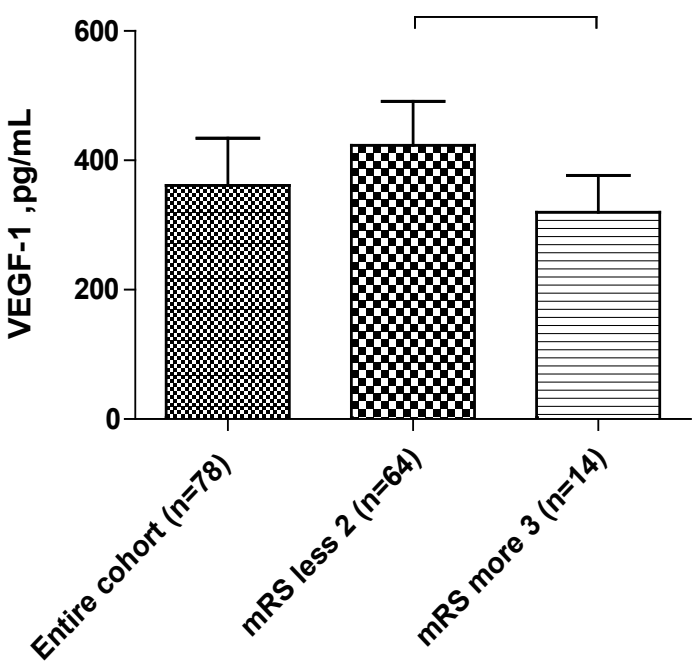

Figure 1: VEGF-1 concentrations in Modified Rankin Score $\geq 3$ vs Modified Rankin Score $\leq 2$ population sub-groups. mRS: Modified Rankin Score.

\begin{tabular}{|c|c|c|c|c|}
\hline \multirow{2}{*}{ Variable } & \multicolumn{2}{|c|}{ Univariable analysis } & \multicolumn{2}{c|}{ Multivariable analysis } \\
\cline { 2 - 5 } & B coefficient & P value & B coefficient & P value \\
\hline BMI & 0.22 & 0.034 & 0.11 & 0.12 \\
\hline VEGF-1 & 0.48 & 0.001 & 0.41 & 0.001 \\
\hline Dyslipemia & 0.54 & 0.001 & 0.40 & 0.042 \\
\hline Type two diabetes mellitus & 0.24 & 0.002 & 0.12 & 0.048 \\
\hline Current smoking status & 0.28 & 0.001 & 0.15 & 0.23 \\
\hline $\begin{array}{c}\text { Past history of ischemic } \\
\text { heart diseases }\end{array}$ & 0.12 & 0.026 & 0.06 & 0.12 \\
\hline $\begin{array}{c}\text { Previous ischemic heart } \\
\text { episode }\end{array}$ & 0.18 & 0.001 & 0.04 & 0.14 \\
\hline
\end{tabular}

Table 2: Age- and sex adjusted univariate and multivariate regression analysis for neurologic recovery defined as Modified Rankin Score $\leq 2$ at 6 month. Note: The multivariate regression model included all variables with $p$ value $<0.2$.

\begin{tabular}{|c|c|c|c|c|}
\hline Variables & OR & $\mathbf{9 5 \%}$ Cl & Wald coefficients & P value \\
\hline $\begin{array}{c}\text { Dyslipidemia (present vs } \\
\text { absent) }\end{array}$ & 1.02 & $1.00-1.05$ & 6.37 & 0.28 \\
\hline VEGF-1 per 125 pg / mL & 0.92 & $0.88-0.98$ & 7.5 & 0.001 \\
\hline $\begin{array}{c}\text { Type two diabetes mellitus } \\
\text { (present vs absent) }\end{array}$ & 1.05 & $1.01-1.08$ & 15.4 & 0.001 \\
\hline
\end{tabular}

Table 3: Multivariate Cox regression analysis for favorable neurologic recovery. Modified Rankin Score $\geq 3$ versus Modified Rankin Score $\leq 2$.

out infection and other inflammatory diseases before sampling, but we cannot exclude that some patients had unrecognized conditions responsible for the elevated VEGF-1 levels observed. However, additional verification of atherosclerosis as well as intracranial artery occlusive disease can be required. We supposed that these limitations might not have a significant influence to study data interpretation.

\section{References}

1. Lackland DT, Roccella EJ, Deutsch AF, Fornage M, George MG, et al. (2014) Factors influencing the decline in stroke mortality: a statement from the American Heart Association/American Stroke Association. Stroke 45: 315-353.

2. Rodriguez CJ, Allison M, Daviglus ML, Isasi CR, Keller C, et al. (2014) Status of cardiovascular disease and stroke in Hispanics/Latinos in the United States: a science advisory from the American Heart Association. Circulation 130: 593-625.
3. Lackland DT, Elkind MS, D'Agostino R, Dhamoon MS, Goff DC, et al. (2012) Inclusion of stroke in cardiovascular risk prediction instruments: a statement for healthcare professionals from the American Heart Association/American Stroke Association. Stroke 43: 1998-2027.

4. Sacco RL, Kasner SE, Broderick JP, Caplan LR, Connors JJ, et al. (2013) An updated definition of stroke for the 21st century: a statement for healthcare professionals from the American Heart Association/American Stroke Association. Stroke 44: 2064-2089.

5. Wijdicks EF, Sheth KN, Carter BS, Greer DM, Kasner SE, et al. (2014) Recommendations for the management of cerebral and cerebellar infarction with swelling: a statement for healthcare professionals from the American Heart Association/American Stroke Association. Stroke 45: 1222-1238.

6. Stanca DM, Mărginean IC, Soriţău O, Mureșanu DF (2015) Plasmatic markers for early diagnostic and treatment decisions in ischemic stroke. J Med Life 8 Spec Issue: 21-25.

7. Asahara T, Takahashi T, Masuda H, Kalka C, Chen D, et al. (1999) VEGF contributes to postnatal neovascularization by mobilizing bone marrow-derived endothelial progenitor cells. EMBO J 18: 3964-3972.

8. Higashi Y, Quevedo HC, Tiwari S, Sukhanov S, Shai SY, et al. (2014) Interaction between insulin-like growth factor-1 and atherosclerosis and vascular aging. Front Horm Res 43: 107-124.

9. Zhang Q, Zhao YH (2014) Therapeutic angiogenesis after ischemic stroke: Chinese medicines, bone marrow stromal cells (BMSCs) and their combinational treatment. Am J Chin Med 42: 61-77.

10. Zachary I (2005) Neuroprotective role of vascular endothelial growth factor signalling mechanisms, biological function, and therapeutic potential Neurosignals 14: 207-221.

11. Howangyin KY, Silvestre JS (2014) Diabetes mellitus and ischemic diseases: molecular mechanisms of vascular repair dysfunction. Arterioscler Thromb Vasc Biol 34: 1126-1135

12. Tovar-Y-Romo LB, Ramírez-Jarquín UN, Lazo-Gómez R, Tapia R (2014) Trophic factors as modulators of motor neuron physiology and survival: implications for ALS therapy. Front Cell Neurosci 8: 61.

13. Berezin AE, Lisovaya OA (2014) Predictive Value of Circulating Vascular Endothelial Growth Factor-1 in Arterial Hypertension Patients. Intern Med S 11: $2-10$.

14. Berezin AE, Lisovaya OA (2014) Predicted value of serial measurements of circulating vascular endothelial growth factor-1 in arterial hypertension patients after acute ischaemic stroke. Cardiovascular Medicine 17: 14-20.

15. Collin C, Wade DT, Davies S, Horne V (1988) The Barthel ADL Index: a reliability study. Int Disabil Stud 10: 61-63.

16. Hacke W, Kaste M, Fieschi C, von Kummer R, Davalos A, et al. (1998) Randomised double-blind placebo-controlled trial of thrombolytic therapy with intravenous alteplase in acute ischaemic stroke (ECASS II). Second EuropeanAustralasian Acute Stroke Study Investigators. Lancet 352: 1245-1251.

17. Brott TG, Halperin JL, Abbara S, Bacharach JM, Barr JD, et al. (2011) 2011ASA ACCF/AHA/AANN/AANS/ACR/ASNR/CNS/SAIP/SCAI/SIR/SNIS/SVM/SVS guideline on the management of patients with extracranial carotid and vertebral artery disease: executive summary. J Neurointerv Surg 3: 100-130.

18. Tarantini L, Barbati G, Cioffi G, McAlister FA, Ezekowitz JA, et al. (2015) Clinical implications of the CKD epidemiology collaboration (CKD-EPI) equation compared with the modification of diet in renal disease (MDRD) study equation for the estimation of renal dysfunction in patients with cardiovascular disease. Intern Emerg Med.

19. Seidler RD, Mulavara AP, Bloomberg JJ, Peters BT (2015) Individual predictors of sensorimotor adaptability. Front Syst Neurosci 9: 100.

20. Menezes KK, Scianni AA, Faria-Fortini I, Avelino PR, Carvalho AC, et al. (2015) Potential predictors of lower extremity impairments in motor coordination of stroke survivors. Eur J Phys Rehabil Med.

21. O'Keefe LM, Conway SE, Czap A, Malchoff CD, Benashski S, et al. (2015) Thyroid hormones and functional outcomes after ischemic stroke. Thyroid Res 8: 9.

22. Koh CL, Pan SL, Jeng JS, Chen BB, Wang YH, et al. (2015) Predicting recovery of voluntary upper extremity movement in subacute stroke patients with severe upper extremity paresis. PLoS One 10: e0126857.

23. Babulal GM, Huskey TN, Roe CM, Goette SA, Connor LT, et al. (2015) Cognitive impairments and mood disruptions negatively impact instrumental activities of daily living performance in the first three months after a first stroke. Top Stroke Rehabil 22: 144-151. 